\title{
Effects of propagation conditions on radar beam-ground interaction: impact on data quality
}

\author{
A. Fornasiero ${ }^{1,2}$, P. P. Alberoni ${ }^{2}$, R. Amorati ${ }^{2}$, L. Ferraris ${ }^{1,3}$, and A. C. Taramasso $^{1,3}$ \\ ${ }^{1}$ CIMA Università di Genova e della Basilicata, Savona, Italy \\ ${ }^{2}$ A.R.P.A Emilia-Romagna - Servizio Idrometeorologico, Bologna, Italy \\ ${ }^{3}$ DIST Università di Genova, Genova, Italy
}

Received: 25 October 2004 - Revised: 11 May 2005 - Accepted: 16 May 2005 - Published: 8 June 2005

\begin{abstract}
A large part of the research in the radar meteorology is devoted to the evaluation of the radar data quality and to the radar data processing. Even when, a set of absolute quality indexes can be produced (like as ground clutter presence, beam blockage rate, distance from radar, etc.), the final product quality has to be determined as a function of the task and of all the processing steps.
\end{abstract}

In this paper the emphasis lies on the estimate of the rainfall at the ground level taking extra care for the correction for ground clutter and beam blockage, that are two main problems affecting radar reflectivity data in complex orography. In this work a combined algorithm is presented that avoids and/or corrects for these two effects. To achieve this existing methods are modified and integrated with the analysis of radar signal propagation in different atmospheric conditions. The atmospheric refractivity profile is retrieved from the nearest in space and time radiosounding. This measured profile is then used to define the 'dynamic map' used as a declutter base-field. Then beam blockage correction is applied to the data at the scan elevations computed from this map.

Two case studies are used to illustrate the proposed algorithm. One is a summer event with anomalous propagation conditions and the other one is a winter event. The new algorithm is compared to a previous method of clutter removal based only on static maps of clear air and vertical reflectivity continuity test. The improvement in rain estimate is evaluated applying statistical analysis and using rain gauges data. The better scores are related mostly to the "optimum" choice of the elevation maps, introduced by the more accurate description of the signal propagation.

Finally, a data quality indicator is introduced as an output of this scheme. This indicator has been obtained from the general scheme, which takes into account all radar data processing steps.

Correspondence to: P. P. Alberoni

(palberoni@smr.arpa.emr.it)
1 The radar beam propagation in standard and anomalous conditions

In the low troposphere, the radar signal trajectory depends on the variation of the refractive index $n$, which is a function of the temperature and water content. Usually, for dimensional reasons, the propagation conditions are described using the refractivity $\left(N=(n-1) \times 10^{6}\right)$. For microwaves in the low troposphere, this parameter can be estimated by the formula of Bean and Dutton (1968):

$N=(77.6 / T) /\left(P+4810 P_{w} / T\right)$

where $N$ is a dimensionless number, $P$ is the total pressure, $P_{w}$ is the partial pressure of water (mbar) and $T$ is the temperature ( ${ }^{\circ}$ Kelvin). Only the firsts kilometres of atmosphere are important for most radar meteorology applications, where the refractivity gradient is approximately $-40 \mathrm{~km}^{-1}$ in standard conditions. In cases of temperature inversion and very humid air conditions, this value can be lower than $-157 \mathrm{~km}^{-1}$, that is a weather condition favourable to anomalous propagation (hereinafter anaprop). The propagation depends strongly on local thermodynamic conditions, which vary substantially in space and time. Anaprop events are generally determined from the thermodynamic conditions in the first 200-300 m of atmosphere. In case of a flat terrain the radar beam reaches this altitude over a short range. As a consequence, even if a sounding station, located close the radar, is unable to characterize the propagation conditions over the whole radar domain, it can be sufficient just to recognize anaprop conditions. In our work we have used the TEMP (WMO radiosoundings data format) of San Pietro Capofiume station, interpolated at steps of $25 \mathrm{~m}$, to derive the refractivity profile, assuming that this approach is valid in the mountains area. Once the gradient of refractivity is known, the path of a wave relative to the earth can be calculated using the formula reported by Doviak and Zrnic (1984).

$h=\sqrt{r^{2}+\left(k_{e} a\right)^{2}+2 r k_{e} a \sin \theta}-k_{e} a+H_{0}$ 
Table 1. Anomalous propagation detection thresholds of the VCT, for the first bin with anaprop in a azimuth and for the sequent bins in the same azimuth ("behind anaprop"). Anomalous propagation is identified when the difference between $\mathrm{Z}$ value at the elevation of dynamic map and at the successive elevation exceeds the threshold $\mathrm{T} 1$, or if this difference is greater than $0 \mathrm{dbZ}$ and the reflectivity value at the successive elevation is lower than $\mathrm{T} 2$.

\begin{tabular}{ccc}
\hline Threshold (dbZ) & Standard & Behind anaprop \\
\hline T1 & 30 & 15 \\
T2 & -10 & 0 \\
\hline
\end{tabular}

where $r$ is the radar range, $a$ the Earth's radius, $\theta$ he antenna elevation and $H_{0}$ the antenna's height; $k_{e} a$ is the effective Earth's radius, which is a function of the refractivity gradient.

\section{Clutter and beam blocking removal}

\subsection{The dynamic map}

The most simple and low cost way to suppress clutter echoes is to use maps of clear air (hereinafter CAM), i.e. to store the reflectivity values from radar scans at different elevation angles taken during clear air weather conditions. This approach is efficient to remove a large part of ground clutter in standard conditions and to suppress side-lobe clutter. However, this approach resolves neither anomalous propagation effects nor radar beam blockage. Therefore, we have modified this method by introducing propagation and beam blockage modelling. As a result two new maps have been defined, namely a forecasted clutter elevation map (hereinafter FCEM) and a beam blocking elevation map (hereinafter BBEM). The first is obtained calculating the path of radar beam using the Eq. (2), and overlaying it to a DEM (digital elevation model). Elevation angles of scans are chosen in each bin to minimize the ground clutter. If the $3-\mathrm{dB}$ beam intercepts even partially the ground, the elevation considered over that cell is increased. In order to identify this condition, the half power beam vertical section is represented as an ensemble of numerous rays and the path of the lowest one is taken into consideration. To describe the form of the beam section it would be necessary a three dimensional model, therefore we have skipped the problem considering directly the cited multi-rays representation

The second map is chosen in order to ensure that at least $50 \%$ of transmitted power reaches each bin. The beam blocking model based on a geometric-optic approach (Bech et al., 2003) is described in Sect. 2.3. This model requires only the knowledge of the DEM and the refractivity gradient.

To describe the atmospheric condition we have used the radiosoundings falling within the twelve hours before and after the case. The refractivity profile is calculated as time linear combination of the two profiles obtained from the radiosoundings. If the radiosounding is absent, the refractivity

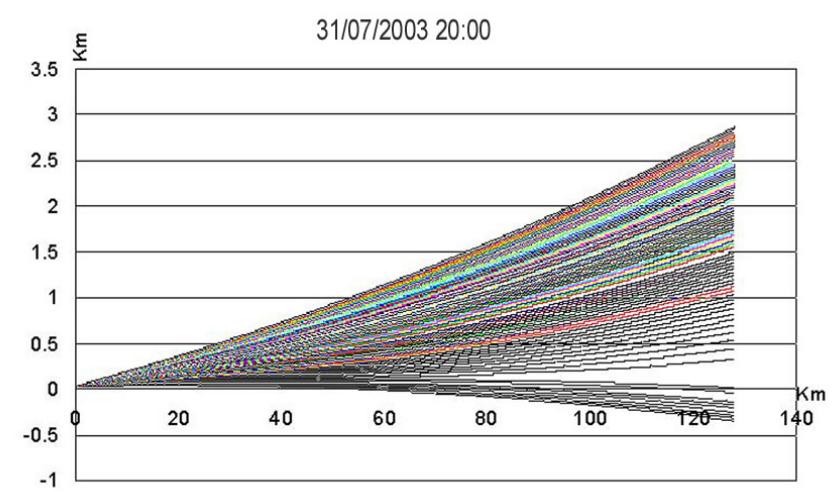

Fig. 1. Beam path at $0.5^{\circ}$ antenna elevation. It has been "broken" the principal lobe in small rays and followed the path of each one.

standard profile is used. Into the dynamic map are stored the highest elevations between those indicated by the three maps.

\subsection{Removal of residual anomalous propagation clutter}

Because of the approximations in the path modelling, such as the spatial homogeny of refractivity profiles and their linear time dependence, it is necessary to remove residual anaprop clutter. The method used in this work has been implemented by Alberoni et al. (2001) and is based on a vertical Z continuity test (hereinafter VCT). Anomalous propagation is identified when the difference between $\mathrm{Z}$ value at the elevation of dynamic map and at the successive elevation exceeds the threshold T1 shown in Table 1, or if this difference is greater than $0 \mathrm{dbZ}$ and the reflectivity value at the successive elevation is lower than T2. The main idea of this methodology is that anaprop clutter, having a small vertical extension, shows, as own "signature", a steep decrease in the produced reflectivity value, more rapid than in cases of rain. We have introduced a modification to this scheme that takes into account cases of rain with limited vertical development. It consists in introducing a condition to apply the test beyond $80 \mathrm{~km}$ from radar: when the elevation is greater than the first, the difference between the reflectivity at the previous and at the chosen elevation must exceed $10 \mathrm{dbZ}$. In this way, the bin is marked as possibly contaminated by anaprop clutter, and only in this case, if the VCT test is positive, it is rejected.

\subsection{Beam blocking model}

The beam blocking model developed from Bech et al. (2003) is based on a geometric-optic approach. Because for typical radar frequencies the physical dimensions of ground targets are much larger than the wavelength, case of scattering of radiowaves from ground can be considered in the geometric optics approximation. This model assumes that, the radar beam has a circular cross-section, where the power density is uniform. Bech et al. (2003) have shown that the difference in terms of beam blockage rate between a Gaussian antenna gain pattern (more realistic in standard conditions) 


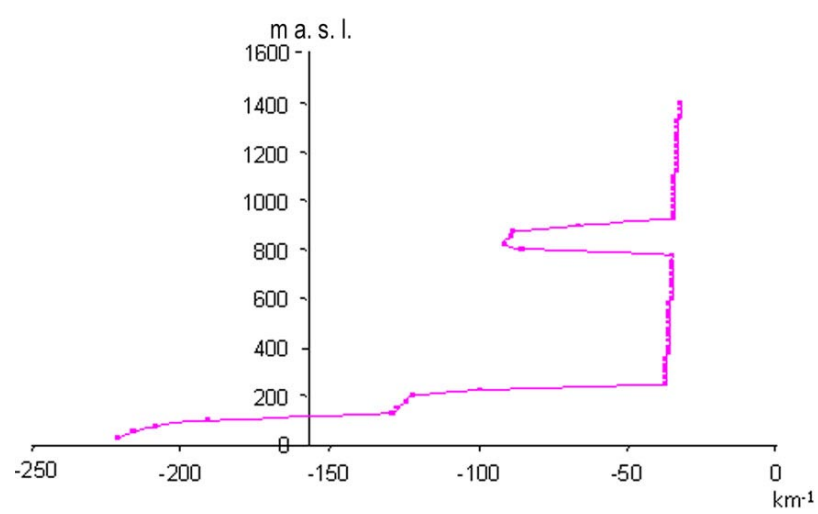

Fig. 2. $N$ gradient profile. Time: 01/08/2003 00:00.

and a uniform circular one is small into the considered beam blockage limits, i.e. 0-50\% (see Fig. 3).

This simplification is useful in nearly all standard propagation conditions. The example in the Fig. 1 shows that the path of the principal lobe "broken" in small rays, at the elevation of 0.5 degree: the lower part bents to the soil and the upper part propagates freely in the atmosphere. We can also conclude that at the elevation of 0.5 degrees, neither the power density could be considered constant, nor the form of the section is circular. However, at the higher elevation angles this approximation is reliable for the application of beam blockage rate evaluation. Therefore, in this case, the fraction of power lost is equal to the fraction of beam cross-section blocked by ground.

2.4 The quality evaluation: a necessary conclusion of data processing

The ground clutter suppression methodology and beam blockage correction can be subdivided into three steps:

1. selection of a dynamic clear air map

2. correction for the power loss

3. removal of anaprop residual clutter using modified VCT method

At the end of this sequence, the quality evaluation synthesizes the information available about the initial datum condition and the processing efficacy.

In a detailed analysis, we can define three levels of quality evaluation each corresponding to an intermediate product:

- the hardware level, where the output quantity is a power

- the measurement level, where the output is reflectivity

- the final product level where the output is the rain rate.

In this work, the input data to our processing scheme is the polar volume. Prior to our processing Doppler clutter suppression was applied to radar signal. Neglecting what happens at the hardware level, we have determined the quality function at the second level.

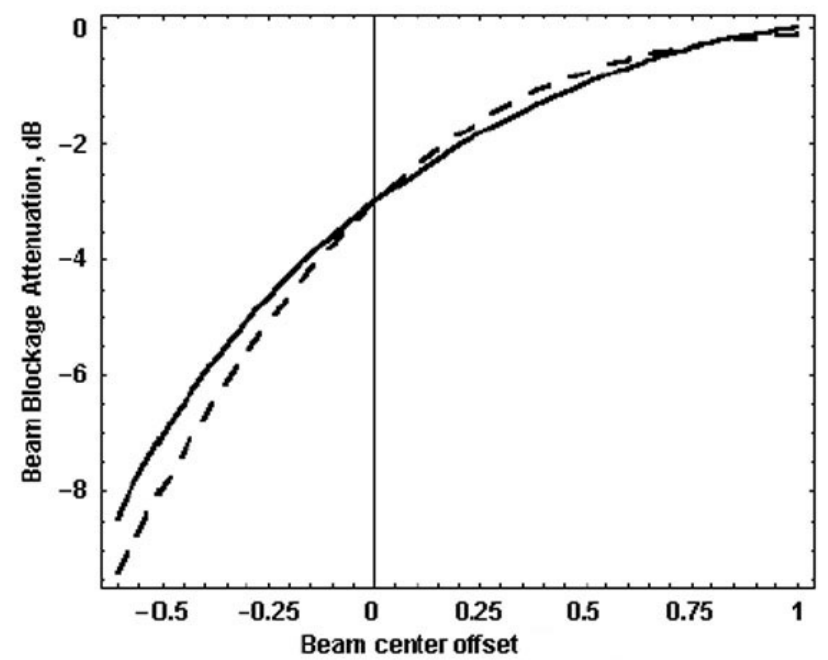

Fig. 3. Comparison between beam blockage attenuation calculated assuming rectangular beam (dashed line) and gaussian beam (solid line). The beam center offset is related to the half-width of a $1.3^{\circ}$ 3-dB beam (figure reported from Bech et al., 2003). The difference between the two assumptions is small taking into consideration a maximum attenuation rate of $50 \%$, i.e. a beam center offset ranging from 0 to 1 (courtesy: AMS).

Assuming that the data quality is determined by $n$ independent factors, that are defined by different steps of radar data processing, we have defined the final quality indicator as a product of $n$ components $Q_{i}(i=1, . . n)$.

$Q=\prod_{1}^{n} Q_{i}^{*}$

Each component is calculated combining the quality indicators of the data before the correction $\left(Q d_{i}\right)$, and after them $\left(Q c_{i}\right)$, taking into account their maxims $Q d_{\max }, Q c_{\max }$

$Q_{i}^{*}=Q_{\max , i}^{*}-\left(Q d_{\max , i}-Q d_{i}\right)\left(Q c_{\max , i}-Q c_{i}\right)$

Assuming the quality values ranging from 0 to 1 the new function is:

$Q_{i}^{*}=1-\left(1-Q d_{i}\right)\left(1-Q c_{i}\right)$

This type of function complies with the following conditions:

a) quality indicator after a correction is higher or at least equal to the one before the correction, because negative values of $Q c$ are rejected;

b) quality indicator after the perfect correction is equal to the one of a perfect data.

The choice of a product function is due to the necessity, to satisfy simultaneously each minimum quality condition to have a reliable data. Furthermore, it is a very general definition that requires the detailed knowledge of the task and of the process to obtain the product. The methodology previously described is a part of the complete chain of data 
Table 2. Quality components. $Q d$ and $Q c$ are the quality of the datum and of the correction respectively. $B B$ and $B B$ max indicates the actual and the maximum accepted beam blocking rate. $\beta$ is the regression exponent of radar-gauges assessment factor, $\theta$ er $r$ is the antenna pointing error, $\Delta T$ and $\Delta R$ are the time scale and the distance scale, $\Delta t_{r s}$ and $\Delta r_{r s}$ are the time and space distance from the nearest radiosounding, $r$ is distance from radar.

\begin{tabular}{lll}
\hline Factors affecting quality & $Q d$ & $Q c$ \\
\hline Beam blocking & $1-B B / B B \max$ & $\mathrm{f}(B B) * \mathrm{f}(\theta e r r) * \mathrm{f}\left(\Delta t_{r s}\right) * \mathrm{f}\left(\Delta r_{r s}\right)$ \\
Clutter & 0 if clutter is present; 1 elsewhere & 0.5 \\
Vertical continuity test & 0.8 if upper elevation is not present; 1 elsewhere & 0 \\
Radar distance & $Q d=\exp (-\beta r)$ & 0 \\
$f(\theta e r r)$ & & $1-\theta$ err \\
$f\left(\Delta t_{r s}\right)$ & & $\exp \left(-\Delta t_{r s} / \Delta T\right)$ \\
$f\left(\Delta r_{r s}\right)$ & $\exp \left(-\Delta r_{r s} / \Delta R\right)$ \\
\hline
\end{tabular}

processing that can include attenuation correction, vertical profile of reflectivity reconstruction, secondary trip echo removal, event classification etc. Each one of these steps produces quality output potentially to be included in the product quality indicator. In this work only a part of this problem is considered, namely only the quality components that are related to the problems considered in the presented methodology: clutter, beam blocking, radar distance, vertical continuity test are considered. These functions are given in Table 2 .

In case of the beam blocking, the data quality before the correction is defined as linear dependent on the beam blockage rate, with a maximum limit of $50 \%$. Above this limit the data is rejected. The quality of the correction is determined as a product of a factor decreasing with beam blocking rate, and depends on the time and space distance from the nearest radiosounding $\left(\Delta t_{r s}\right.$ and $\left.\Delta r_{r s}\right)$ and on the antenna pointing error, $\theta e r r$. For the radiosounding distance we have considered exponential degradation with a time scale $\Delta T$ of $4 \mathrm{~h}$ and a distance scale $\Delta R$ of $50 \mathrm{~km}$ (this values are derived from meteorological data assimilation common procedures). The last factor depends linearly on the antenna pointing error $\theta$ err: the function is derived from the work of Bech et al. (2003), that shows that an errors of $0.05^{\circ}$ and $0.1^{\circ}$ produce an error of $5 \%$ and $10 \%$ respectively in the beam blockage evaluation.

In case of clutter suppression, the quality before the correction is assumed constant and equal to 0 if clutter is detected and 1 elsewhere. The quality of the correction is assumed equal to 0.5 .

In case of vertical continuity test presence, the quality of the datum is assumed equal to 0.8 if the test is not applied (i.e. it falls the upper elevation datum), and 1 elsewhere.

Finally it has been chosen a quality component decreasing exponentially with the radar distance (see Table 2) his definition is derived from the work of Koistinen and Puhakka (1981) where the climatological assessment factor (ratio between rain gauges rain and radar rain) is shown to have an exponential degradation vs. distance. The regression coefficient $\beta$ is calculated using one year of data and used in the quality component function. This value partially includes the climatological effect of the vertical profile of reflectivity (VPR), because it is related to the increase of the height. The choice of the component functions is reasonable but requires optimization for operational applications.

\section{Results}

The described methodology has been applied and tested on two case studies related to different types of meteorological events. The first is a thunderstorm occurred in summer 2003 , on 31 July when anomalous propagation conditions have been verified. The second is a case of stratiform rain occurred in winter 2003, on 10 December in the afternoon. The reflectivity dataset is generated by San Pietro Capofiume radar located in Italy, in the Po Valley $30-40 \mathrm{~km}$ from the mountains of Appennino. A radiosounding station is colocated with the radar. We have used the radar scans at min $00,15,30,45$, decluttered by Doppler filter and acquired at PRF of $1200 \mathrm{~Hz}$ and impulse time of $0.5 \mu$ s, i.e. with a range resolution of $75 \mathrm{~m}$, smoothed afterwards to $250 \mathrm{~m}$. The antenna beamwidth is $0.9^{\circ}$ and their scan elevations are $0.5^{\circ}$, $1.4^{\circ}, 2.3^{\circ}, 3.2^{\circ}, 4.1^{\circ}$. The performance of the new algorithm has been evaluated, at first qualitatively, comparing it with a method of reflectivity correction based only on CAM and VCT. Thereafter a statistical analysis on the two complete events has been performed to evaluate quantitatively its reliability. To illustrate the operational sequence including the quality output we have chosen an instant of the thunderstorm event with anomalous propagation. In Fig. 2 the refractivity gradient obtained from the nearest radiosounding is presented. A deep temperature inversion caused anaprop in the first $200 \mathrm{~m}$, in fact the value of $\mathrm{dN} / \mathrm{dh}$ is smaller than $-157 \mathrm{~km}^{-1}$. The form of the radar beam for the elevation of $0.5^{\circ}$ is illustrated in Fig. 1 and has been previously discussed. Using this atmospheric description we have defined the FCEM and the BBEM and combining them with the CAM we have obtained the dynamic map (Fig. 4). In this case, because of the strong anomalous propagation, the elevation is nearly overall greater than the first. At this elevation the modelled beam blockage is almost absent. 

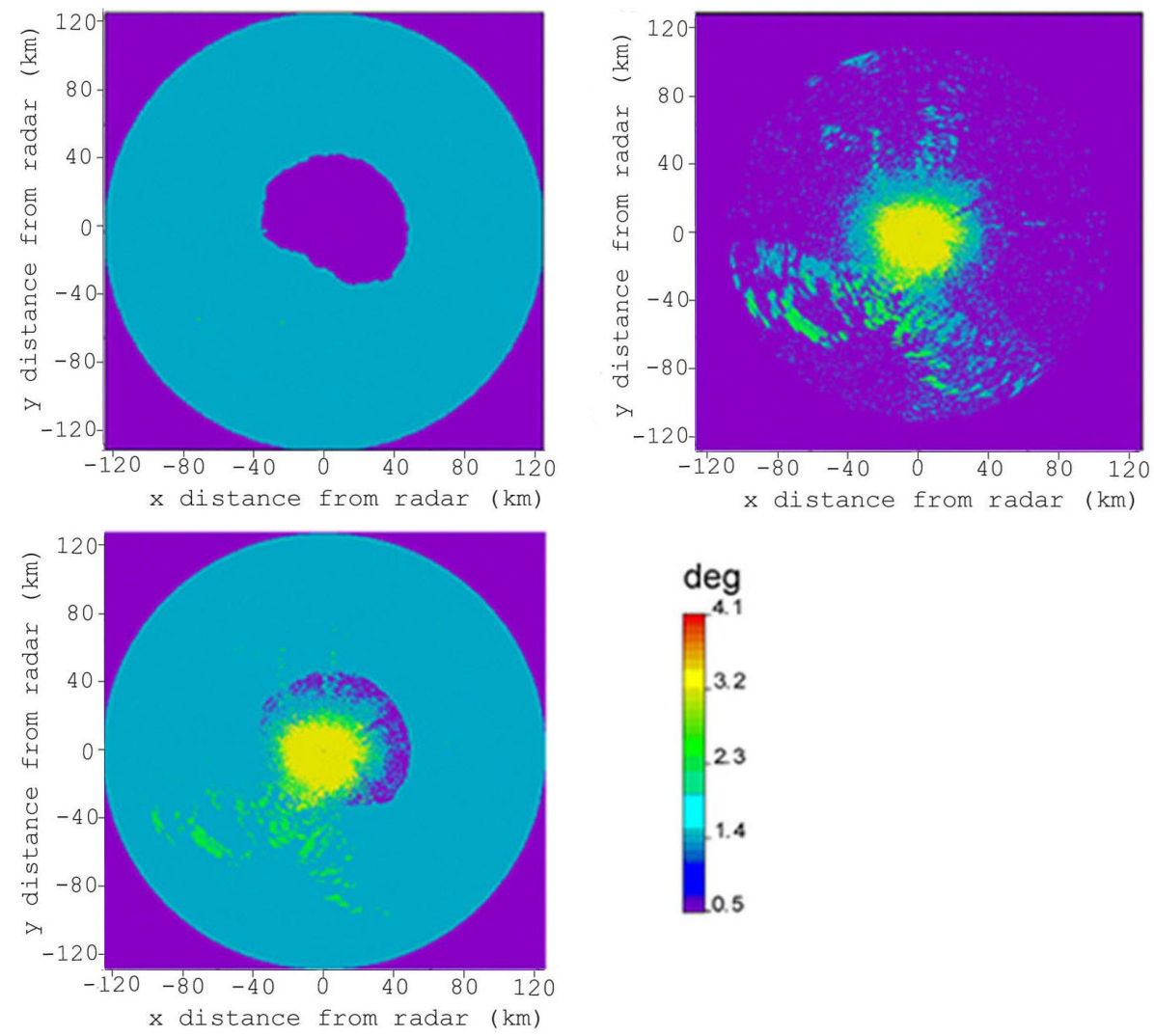

Fig. 4. High panel: BBEM+FCEM (lefts) and static map of clear air CAM (rights). Low panel: final dynamic map. Time: 31/07/2003 20:00.
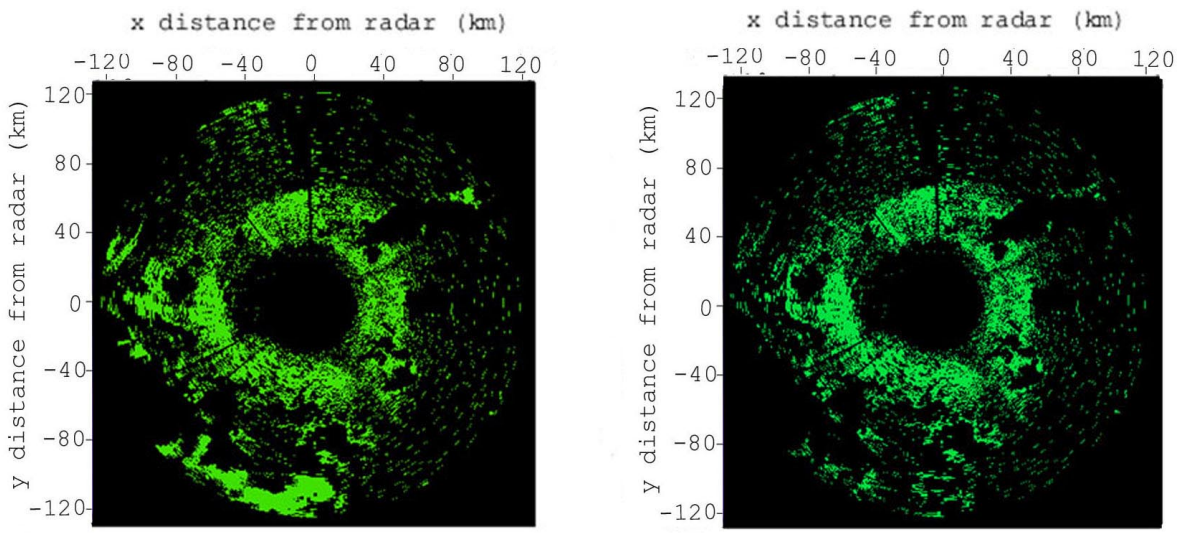

Fig. 5. Anomalous propagation (green), detected from original scheme of Alberoni (left panel) and from new scheme (right panel). Time: 31/07/2003 20:00

Next step is the removal of anomalous propagation clutter. In Fig. 5 the maps of anaprop obtained from original and modified scheme are presented: at the SO bound of the map a precipitation echo is recognised from the original algorithm as anomalous propagation; the second scheme avoids this error.

Thereafter the set of quality indexes (i.e. the fractional beam blocking, and the anomalous propagation clutter detection) has been calculated and used for the evaluation of the "final" quality, following the method described in Sect. 2.4 and in Table 2. In Fig. 6, the map of data quality calculated cell per cell is represented, for the two compared methods and in a Cartesian grid of $1 \mathrm{~km} \times 1 \mathrm{~km}$ of resolution. It is visible the high quality difference behind the mountains (zone between $180^{\circ}$ and $270^{\circ}$ azimuth, affected by beam blockage) and in the anaprop area. Further in the first $20-25 \mathrm{~km}$ the effect of side-lobe clutter contamination is clearly visible. Inside this area, data are not corrected by the methods; indeed in this zone it is used the higher elevation available, since that also this one is still affected by some clutter residual and so 

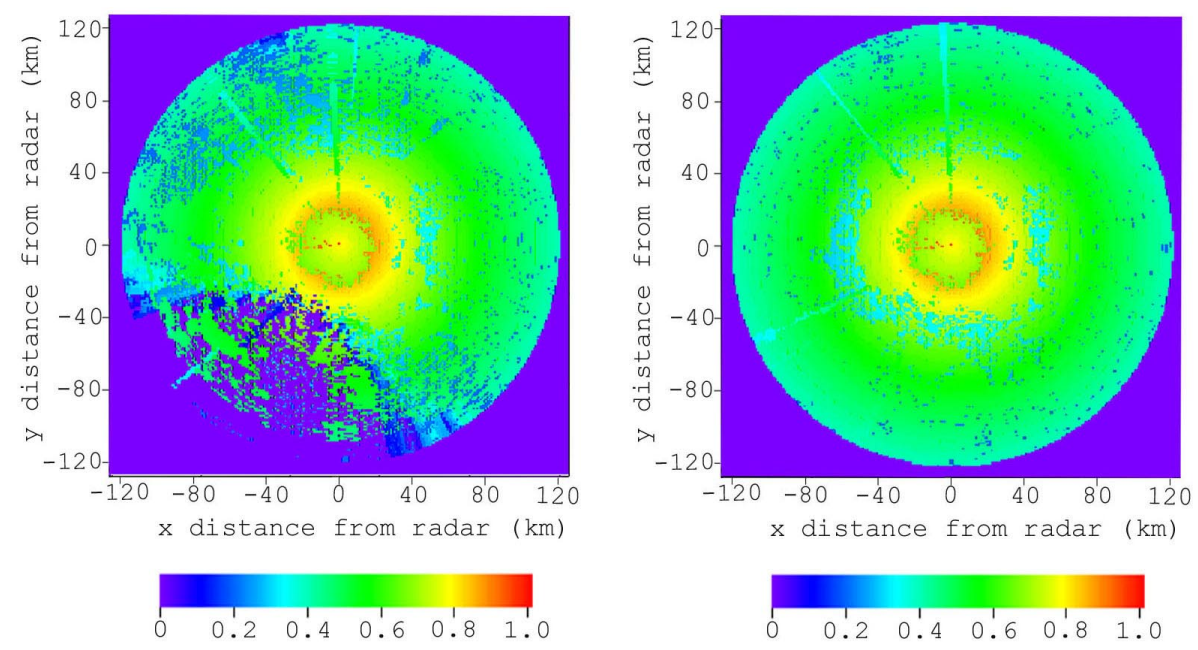

Fig. 6. Quality maps, in cartesin grid, related to the CAM+VCT method (left panel) and to the new method of radar data correction (right panel). Time: 31/07/2003 20:00.

it is not possible to apply the vertical continuity test. As a result the quality is lower than elsewhere. Furthermore, the average quality value increases (using the new methodology) from 0.49 to 0.55 .

Once we have illustrated all the processing steps, let us focus the attention on the events. The cumulated rain for the stratiform event has been calculated, using a medium Marshall and Palmer relation. Figure 7 shows the reflectivity map at $1 \mathrm{~km}$ resolution obtained from the method of clutter removal based on CAM and VCT, and that obtained from the new combined method. It is clear that the new scheme reduces the ground clutter and the beam blockage effect (beyond the mountains the signal is more continuous). It is also visible that the remaining problem of the vertical variation of reflectivity has a strong affect on the final result.

To highlight the impacts of the new algorithm inside and beyond the mountains area, a quantitative-statistical analysis has been performed using hourly rain measured during the event from about 130 raingauges located from $135^{\circ}$ to $270^{\circ}$ azimuth and more than $20 \mathrm{~km}$ far from radar, to avoid the area of secondary lobes. The radar rain has been interpolated in a grid of $1 \mathrm{~km} \times 1 \mathrm{~km}$ of resolution and cumulated in the hour; the hourly datum of each raingauge has been compared with them of the radar in the correspondent cell. To convert $Z$ in rain $(R)$, we have used an equation of type Marshall and Palmer (1948), $Z=a R^{b}$. In absence of the optimum pair of coefficients $a$ and $b$ it has been fixed $a=250$ and $b=1.5$ for stratiform rain and $a=500$ and $b=1.5$ for convective rain as indicated by Joss and Waldvogel (1970). Calling $R_{G}$ the hourly cumulated rain rate measured by the gauges and $R_{R}$ them measured by the radar over the correspondent cells, the bias $\left\langle\varepsilon_{R}\right\rangle$ is defined as

$$
\left\langle\varepsilon_{R}\right\rangle=\left\langle R_{R}-R_{G}\right\rangle
$$

where the angle brackets means the average over time and over the cells. The other calculated indexes are (Marzano et al., 2004):

- the root mean square error

$R M S E=\sqrt{\left\langle\varepsilon_{R}^{2}\right\rangle}$

- the fractional mean reduction

$F M R=\frac{\left\langle R_{G}\right\rangle-\left\langle\varepsilon_{R}\right\rangle}{\left\langle R_{G}\right\rangle}$

- the fractional variance reduction

$F V R=\frac{\left\langle\sigma_{R_{P}}^{2}\right\rangle-\left\langle\sigma_{\varepsilon_{E}}^{2}\right\rangle}{\left\langle\sigma_{R_{P}}^{2}\right\rangle}$

The optimal value of FMR, FVR is 1 and of RMSE is 0 . We have calculated this indexes using radar rain rates obtained from the new algorithm (BDA) and from "CAM+VCT" algorithm (SA) and we have compared them. Figures 8 and 9 shows RMSE and the FMR, for the two methods, calculated respectively in the summer and in the winter case study.

The new algorithm shows a lower RMSE in both cases. It is difficult to evaluate the performance of the algorithms observing the bias and similar indexes as FMR. This indexes are strictly influenced by factors such as the correct calibration of $a$ and $b$ coefficients and the VPR correction. In fact, the introduction of propagation and BB model increases often the height at which the reflectivity data are kept, with respect to the old algorithm; this can imply for the final rain rate a smoothing of the previous improvement or even a worsening.

FVR, in the analysed cases, is very near to 1 because the variance of the error is smaller than the variance of the rain field. Further, it must be noted that the indexes are calculated on cells that are concentrated in the Appennino area into the region Emilia Romagna; i.e. the performance of the algorithm in the boundary of the radar map is also neglected. 

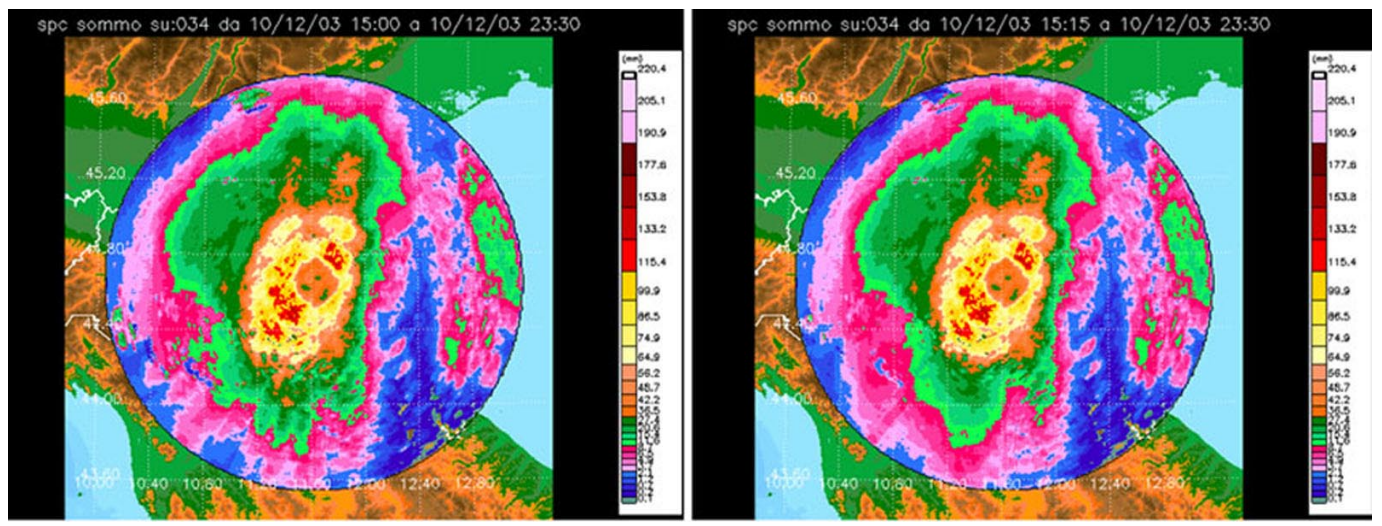

Fig. 7. Map of cumulated rain after CAM+VCT method (left panel) and after the new method correction (right panel). Event 10/12/2003 from 15:00 to 23:00. Increasing rain from blue to red.
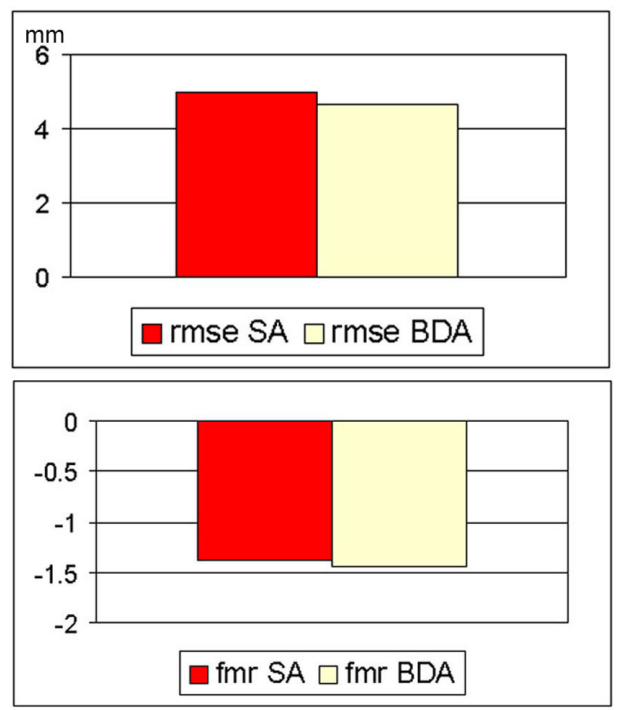

Fig. 8. RMSE and FMR of summer event for the CAM+VCT algorithm ( $\mathrm{SA}=$ static map +anaprop removal) and for the new algorithm (BDA=blocking + dynamic map + anaprop removal). MP coefficients are $a=500, b=1.5$.

\section{Conclusions}

The base-idea of the illustrated methodology is to privilege errors minimization respect to their correction. Anaprop suppression can, in fact, lead to underestimation and beam blocking correction, without adequate knowledge of the atmospheric conditions, can produce wrong results. The presented approach shows some advantages with respect to the CAM+VCT algorithm. Firstly, it takes into account the real (or approximated) atmospheric state and recognizes anomalous propagation conditions. This permits to change the elevations at which the data are kept, avoiding or reducing this artefact, before the application of the anaprop removal algorithm. Second, it introduces the beam blockage correction and produces a more reliable field after the mountains, reduc-
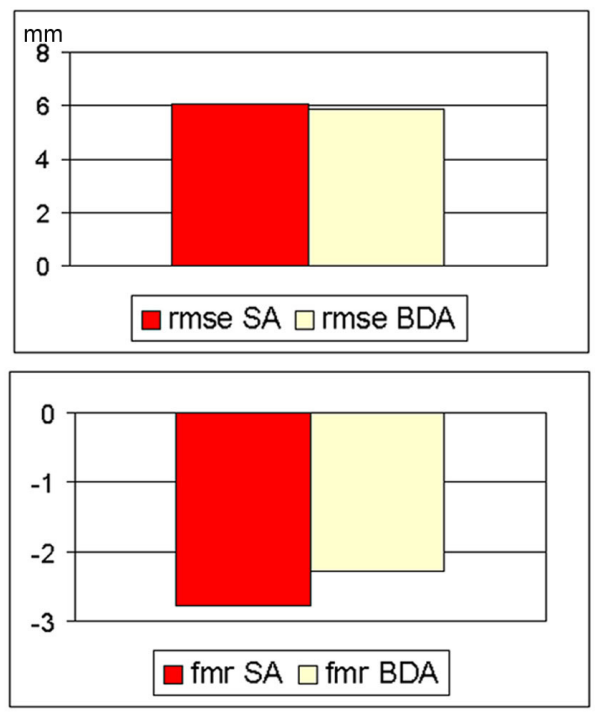

Fig. 9. RMSE and FMR of winter event for the CAM+VCT algorithm ( $\mathrm{SA}=$ static map + anaprop removal) and for the new algorithm (BDA=blocking + dynamic map + anaprop removal). MP coefficients are $a=250, b=1.5$.

ing the shadowing effect of mountains and valleys. Further, the algorithm is simple to implement and has a low computational cost. The schema is suitable to an operational use but it must be integrated with the correction of the vertical profile of reflectivity and with the event classification to choose the $Z-R$ relation. Moreover additional effort should be devoted to verify the efficacy of each processing step and to develop a method to retrieve the approximated propagation conditions in real time.

The other relevant aspect is that, the schema produces indicators of data quality that are useful to evaluate its reliability in hydrological and meteorological applications such as data assimilation. The final quality value takes memory of every correction and processing step and corresponds to the spatial distribution of its effects magnitude. It is closely 
dependent on the problem (i.e. on the product) and on the operation chain to obtain the product. It is meant as tool to evaluate the reliability of radar data for application such as data assimilation, radar composite etc. and can be considered useful to evaluate and compare performances of different algorithms or processing lines, alternatively to the use of rain gauges. However, the quality indicator here presented is only partial. It is necessary to add quality components related to the correction steps here not yet developed (VPR, attenuation, event classification) to obtain a complete description of the data quality. The highest interest is in fact devoted to the evaluation of the quality of the final product. Furthermore, it is necessary to optimise the definition of the quality functions testing them on operational applications and historical dataset.

Acknowledgements. The authors greatly acknowledge J. Bech (MeteoCAT) for the ray propagation and beam blocking code. This work is partially supported by CARPE DIEM, a research project supported by the European Commission under the 5th FP (Contract No. EVG1-CT-2001-0045), and form the GNDCI through the project RAM. The comments and suggestion of the two anonymous referees have been greatly appreciated.

Edited by: G. Boni

Reviewed by: anonymous referees

\section{References}

Alberoni, P. P., Anderson, T., Mezzasalma, P., Michelson, D. B., and Nanni, S.: Use of the vertical reflectivity profile for identification of anomalous propagation, Meteorological Applications, 8, 257266, 2001.

Bean, B. R. and Dutton, E. J.: Radio Meteorology, Dover Publications, 435 pp., 1968.

Bech, J., Codina, B., Lorente, J., and Bebbington, D.: The sensitivity of single polarization weather radar beam blockage correction to variability in the vertical refractivity gradient, Journal of Atmospheric and Oceanic Technology, 20, 6, 845-855, 2003,

Doviak, R. J. and Zrnic, D. S.: Doppler radar and weather observations, Academic Press, 562 pp., 1993.

Joss, J. and Waldvogel, A.: A method to improve the accuracy of radar-measured amounts of precipitation, Prepr. 14th Conf. Radar Meteorol., pp. 237-238, 2001.

Koistinen, J. and Pohjola H.: Operational vertical reflectivity profile correction in radar network composites in Finland, Preprints, 2nd European Radar Conference, ERAD, 2002.

Marzano, F. S., Picciotti, E., and Vulpiani, G.: Rain Field and Reflectivity Vertical Profile Reconstruction From C-Band Radar Volumetric Data, Ieee transactions on geoscience and remote sensing, 42, 5, 2004.

Marshall, J. S and Palmer, W. McK.: The distribution of raindrops with size, J. Meteor., 5, 165-166, 1948.

WMO Publications: Catalogue of Meteorological Bulletins Weather reporting, No. 9, Vol. C1, http://www.wmo.int/web/ ddbs/Jen/VolumeC1/VolC1.html, 2004. 